TRANSACTIONS OF THE

AMERICAN MATHEMATICAL SOCIETY

Volume 357, Number 5, Pages 1963-1975

S 0002-9947(04)03770-5

Article electronically published on October 7, 2004

\title{
TORIC RESIDUE AND COMBINATORIAL DEGREE
}

\author{
IVAN SOPROUNOV
}

\begin{abstract}
Consider an $n$-dimensional projective toric variety $X$ defined by a convex lattice polytope $P$. David Cox introduced the toric residue map given by a collection of $n+1$ divisors $\left(Z_{0}, \ldots, Z_{n}\right)$ on $X$. In the case when the $Z_{i}$ are $\mathbb{T}$-invariant divisors whose sum is $X \backslash \mathbb{T}$, the toric residue map is the multiplication by an integer number. We show that this number is the degree of a certain map from the boundary of the polytope $P$ to the boundary of a simplex. This degree can be computed combinatorially. We also study radical monomial ideals $I$ of the homogeneous coordinate ring of $X$. We give a necessary and sufficient condition for a homogeneous polynomial of semiample degree to belong to $I$ in terms of geometry of toric varieties and combinatorics of fans. Both results have applications to the problem of constructing an element of residue one for semiample degrees.
\end{abstract}

\section{INTRODUCTION}

Toric residue is defined for every collection of $n+1$ divisors on an $n$-dimensional complete toric variety as long as they do not have a common point. It appears in a variety of contexts, e.g. in mirror symmetry [BM] or in sparse polynomial systems [CaD], CDS]. Toric residues were first introduced by D. Cox in [C2] in the case when the divisors are ample and linearly equivalent. In [CCD Cattani, Cox, and Dickenstein extended the definition to the general case and revealed a connection between the toric residue and the sum of local Grothendieck residues in the torus. They also provided an algorithm for computing the toric residue when the divisors are ample using Gröbner bases. Another approach was taken by D'Andrea and Khetan in $\mathrm{AK}$, where they compute the toric residue as a quotient of two determinants. One of the key ideas in both methods is reduction to a particular choice of $n+1 \mathbb{T}$-invariant divisors for which the toric residue is one. This motivates the problem of computing the toric residue for an arbitrary choice of $n+1 \mathbb{T}$-invariant divisors.

Let $X$ be a projective toric variety of dimension $n$ defined by a convex lattice polytope $P$. Consider $n+1$ effective $\mathbb{T}$-invariant divisors $Z_{0}, \ldots, Z_{n}$ on $X$ whose sum $Z$ equals $X \backslash \mathbb{T}$ (or more generally the support of $Z$ equals $X \backslash \mathbb{T}$ ). Then the toric residue map for $Z_{0}, \ldots, Z_{n}$ has a nice combinatorial description: Notice that the irreducible components of the $Z_{i}$ correspond to the facets of the polytope $P$. Thus, we get a "coloring" of the facets of $P$ into $n+1$ colors: a facet has color $i$ if the corresponding irreducible divisor appears in $Z_{i}$. Let us now pick a color $0 \leq i \leq n$

Received by the editors October 19, 2003.

2000 Mathematics Subject Classification. Primary 14M25; Secondary 52B20.

Key words and phrases. Toric residues, combinatorial degree, toric variety, homogeneous coordinate ring, semiample degree. 
for each facet of the $n+1$ facets of the standard $n$-simplex $\Delta$, so that all facets have different colors. Choose a continuous piecewise linear map $f: \partial P \rightarrow \partial \Delta$ that matches the colors. Such a map exists as long as $Z_{0} \cap \cdots \cap Z_{n}$ is empty. The degree of $f$ is independent of the choice of $f$ and is called the combinatorial degree of the coloring of $P$ (we give the precise definition in Section 1). We prove that the toric residue for $Z_{0}, \ldots, Z_{n}$ coincides with the combinatorial degree of the coloring defined by the $Z_{i}$ (Theorem 2.2, Theorem 2.3). The combinatorial degree can be computed explicitly as a signed number of certain complete flags of faces of $P$ (see Theorem 1.7).

In the second part of the paper we consider a radical monomial ideal $I$ of the homogeneous coordinate ring $S=\bigoplus_{\alpha \in A_{n-1}(X)} S_{\alpha}$ of $X$ (see [C1]). Let $z_{1}, \ldots, z_{m}$ be the minimal generating set of $I$. Consider a homogeneous polynomial $F \in S_{\alpha}$ of semiample degree $\alpha$ (i.e. the line bundles corresponding to $\alpha$ are generated by global sections). It follows from [CCD, Section 2, that if $\alpha$ is $\mathbb{Q}$-ample the polynomial $F$ belongs to $I$ as long as $Z_{1} \cap \cdots \cap Z_{m}$ is empty, where $Z_{i}$ is the zero set of $z_{i}$ on $X$. In Theorem 3.3 we extend this observation. It is known that if $\alpha$ is semiample, there exists a unique toric variety $X^{\prime}$ and a surjective morphism $\pi: X \rightarrow X^{\prime}$ such that $\alpha$ is the pull-back of an ample class on $X^{\prime}$ (see [M], Theorem 1.2). We show that a generic polynomial $F$ of semiample degree lies in $I$ if and only if $\pi\left(Z_{1}\right) \cap \cdots \cap \pi\left(Z_{m}\right)$ is empty on $X^{\prime}$. This condition also has a combinatorial interpretation (Theorem 3.3 part (4)).

Let us remark that it is still an open question of how to compute the toric residue map for arbitrary non-ample divisors even when we know that the map is an isomorphism (e.g. for big and nef divisors). In the final section we apply our results to this problem. We obtain a combinatorial condition when it is possible to construct an element of toric residue one. More results in this direction are obtained in the joint work with A. Khetan [KS.

\section{Combinatorial degree}

1.1. Polyhedral sets. A polyhedral set $X$ is a finite union of convex compact polyhedra intersecting in faces. We assume that they all are embedded in some Euclidean space $E$, thus $X$ is a topological space with topology inherited from $E$. The dimension of $X$ is the maximum of dimensions of the polyhedra it contains.

Example 1. Boundary subdivision. Let $P \subset E$ be a convex $n$-dimensional polytope. Then the boundary $\partial P$ is a polyhedral set of dimension $n-1$. More generally, any polyhedral subdivision of $\partial P$ is a polyhedral set of dimension $n-1$.

For the purposes of this paper it is enough to consider only polyhedral sets of Example 1.

Let $X$ be a polyhedral set and let $\mathcal{F}(X)$ be the set of all faces of all polyhedra appearing in $X$. The set $\mathcal{F}(X)$ is a finite partially ordered set by inclusion.

Definition 1.1. Let $X, Y$ be two polyhedral sets and let $\psi: \mathcal{F}(X) \rightarrow \mathcal{F}(Y)$ be a map that preserves the partial ordering. A continuous map $f_{\psi}: X \rightarrow Y$ is called a characteristic map for $\psi$ if the image of every face $G \in \mathcal{F}(X)$ under $f_{\psi}$ lies in $\psi(G)$.

For any map $\psi: \mathcal{F}(X) \rightarrow \mathcal{F}(Y)$ there exists a characteristic map $f_{\psi}: X \rightarrow Y$. One can construct a piecewise linear $f_{\psi}$ using barycentric subdivisions of $X$ and $Y$ (see [], Proposition 2.1). 
It is easy to see that if $f_{\psi}$ and $f_{\psi}^{\prime}$ are two characteristic maps for $\psi$, then for any $0 \leq t \leq 1$ the map $f_{\psi}^{t}=(1-t) f_{\psi}+t f_{\psi}^{\prime}$ is also characteristic for $\psi$.

Definition 1.2. Let $X$ and $Y$ be $n$-dimensional polyhedral sets and let $\psi: \mathcal{F}(X) \rightarrow$ $\mathcal{F}(Y)$ be a map that preserves the partial ordering. Let $f_{\psi}$ be any characteristic map for $\psi$. The induced map of the $n$-th homology groups

$$
H_{n}\left(f_{\psi}\right): H_{n}(X) \rightarrow H_{n}(Y)
$$

is called the combinatorial degree map of $\psi$.

By the above the combinatorial degree map is independent of the choice of a characteristic map $f_{\psi}$. We denote it by c.deg $\psi$.

Next we will see that the combinatorial degree map is invariant under subdivisions of $X$.

Definition 1.3. A polyhedral set $X^{\prime}$ is called a subdivision of a polyhedral set $X$ if every polyhedron in $X$ is a finite union of polyhedra in $X^{\prime}$, and the support of $X$ equals the support of $X^{\prime}$. We say that $\psi^{\prime}: \mathcal{F}\left(X^{\prime}\right) \rightarrow \mathcal{F}(Y)$ refines $\psi: \mathcal{F}(X) \rightarrow$ $\mathcal{F}(Y)$ if $G^{\prime} \subset G$ implies $\psi^{\prime}\left(G^{\prime}\right) \subset \psi(G)$ for every $G^{\prime} \in \mathcal{F}\left(X^{\prime}\right)$ and $G \in \mathcal{F}(X)$.

Proposition 1.4. Let $X$ and $Y$ be n-dimensional polyhedral sets. Let $X^{\prime}$ be a subdivision of $X$. Consider $\psi: \mathcal{F}(X) \rightarrow \mathcal{F}(Y)$ and $\psi^{\prime}: \mathcal{F}\left(X^{\prime}\right) \rightarrow \mathcal{F}(Y)$ that refines $\psi$. Then c.deg $\psi^{\prime}=$ c.deg $\psi$.

Proof. Let $f_{\psi^{\prime}}: X^{\prime} \rightarrow Y$ be any characteristic map for $\psi^{\prime}$. Since $\psi^{\prime}$ refines $\psi$ the map $f_{\psi^{\prime}}$ considered as a map from $X$ to $Y$ is characteristic for $\psi$. Therefore c.deg $\psi^{\prime}=H_{n}\left(f_{\psi^{\prime}}\right)=$ c.deg $\psi$.

1.2. Combinatorial degree for polytopes. Now assume that $X$ and $Y$ are boundary subdivisions of some convex $n$-dimensional polytopes $P$ and $Q$ as in Example 1 In this case we have isomorphisms $H_{n-1}(X) \cong \mathbb{Z}$ and $H_{n-1}(Y) \cong \mathbb{Z}$ given by a choice of orientations of $P$ and $Q$. The map (1.1) is then the multiplication by an integer number. This number will be called the combinatorial degree of $\psi$ and denoted also by c.deg $\psi$.

Remark 1.5. The combinatorial degree is a global analog of combinatorial coefficients, first introduced by O. Gelfond and A. Khovanskii in GKh1. Combinatorial coefficients appear in the Gelfond-Khovanskii residue formula and the Khovanskii product of roots formula for a polynomial system whose Newton polytopes have generic relative position (see [GKh2] and [Kh] for details).

The next theorem shows how to compute the combinatorial degree for polytopes. We will need the following definition.

Definition 1.6. Let $X$ be a boundary subdivision of an $n$-dimensional convex oriented polytope $P$. Consider a complete flag in $X$ (a maximal chain of elements of $\mathcal{F}(X))$ :

$$
\mathcal{X}: X_{0} \subset \cdots \subset X_{n-1}, \quad \operatorname{dim} X_{i}=i .
$$

For every $1 \leq i \leq n$ choose a vector $e_{i}$ which begins at $X_{0}$ and points strictly inside $X_{i}$, where $X_{n}=P$. Define the sign of the flag $\mathcal{X}$ to be 1 if $\left(e_{1}, \ldots, e_{n}\right)$ gives a positive oriented frame for $P$, and -1 otherwise. 
Let $X$ and $Y$ be boundary subdivisions of $n$-dimensional convex oriented polytopes $P$ and $Q$, and $\psi: \mathcal{F}(X) \rightarrow \mathcal{F}(Y)$. For complete flags $\mathcal{X}: X_{0} \subset \cdots \subset X_{n-1}$ in $X$ and $\mathcal{Y}: Y_{0} \subset \cdots \subset Y_{n-1}$ in $Y$ we will write $\psi(\mathcal{X})=\mathcal{Y}$ if and only if $\psi\left(X_{i}\right)=Y_{i}$ for all $0 \leq i \leq n-1$.

Theorem $1.7([\mathbf{S}])$. Fix any complete flag $\mathcal{Y}$ in $Y$. Then the combinatorial degree of $\psi$ is equal to the sign of $\mathcal{Y}$ times the number of all complete flags $\mathcal{X}$ in $X$ counted with signs, such that $\psi(\mathcal{X})=\mathcal{Y}$ :

$$
\operatorname{c.deg} \psi=\operatorname{sgn} \mathcal{Y} \sum_{\psi(\mathcal{X})=\mathcal{Y}} \operatorname{sgn} \mathcal{X} .
$$

Now let $P$ be an $n$-dimensional convex polytope in $E, \operatorname{dim} E=n$, and consider its polar polytope $P^{\circ}$ in the dual space $E^{*}$. Let $X=\partial P$ and $X^{*}=\partial\left(P^{\circ}\right)$. Recall that there is a one-to-one and order-reversing correspondence $G \mapsto G^{*}$ between $\mathcal{F}(X)$ and $\mathcal{F}\left(X^{*}\right)$ such that $\operatorname{dim} G^{*}=n-1-\operatorname{dim} G$.

Definition 1.8. Let $X=\partial P, Y=\partial Q$ for convex $n$-dimensional polytopes $P$ and $Q$ in $E$. Let $\psi: \mathcal{F}(X) \rightarrow \mathcal{F}(Y)$ be a map of partially ordered sets. Define the dual map $\psi^{*}: \mathcal{F}\left(X^{*}\right) \rightarrow \mathcal{F}\left(Y^{*}\right)$ by putting $\psi^{*}\left(G^{*}\right)=(\psi(G))^{*}$ for any $G \in \mathcal{F}(X)$.

It is readily seen that $\psi^{*}$ preserves the partial order.

Proposition 1.9. Let $P, Q$ be convex n-dimensional polytopes in $E$, and $X=\partial P$, $Y=\partial Q$. Consider any $\psi: \mathcal{F}(X) \rightarrow \mathcal{F}(Y)$ and its dual $\psi^{*}: \mathcal{F}\left(X^{*}\right) \rightarrow \mathcal{F}\left(Y^{*}\right)$. Then $\mathrm{c} . \operatorname{deg} \psi=\mathrm{c} . \operatorname{deg} \psi^{*}$.

Proof. This follows from Theorem 1.7 Indeed, every complete flag $\mathcal{X}$ in $X$ corresponds to a unique complete flag $\mathcal{X}^{*}$ in $X^{*}$ and $\operatorname{sgn} \mathcal{X}=\lambda \operatorname{sgn} \mathcal{X}^{*}$, where $\lambda= \pm 1$ and is the same for all $\mathcal{X} 1$ Then for any fixed flag $\mathcal{Y}$ in $Y$

$$
\operatorname{c.deg} \psi=\operatorname{sgn} \mathcal{Y} \sum_{\psi(\mathcal{X})=\mathcal{Y}} \operatorname{sgn} \mathcal{X}=\lambda \operatorname{sgn} \mathcal{Y}^{*} \sum_{\psi^{*}\left(\mathcal{X}^{*}\right)=\mathcal{Y}^{*}} \lambda \operatorname{sgn} \mathcal{X}^{*}=\operatorname{c.deg} \psi^{*} .
$$

1.3. Simplicial coloring. Consider a convex $n$-dimensional polytope $P$.

Definition 1.10. Let $C_{0}, \ldots, C_{n}$ be closed subsets of $\partial P$, each set being the union of some facets of $P$. We say that $C=\left(C_{0}, \ldots, C_{n}\right)$ forms a simplicial coloring of (the boundary of) $P$ if

(1) $C_{0} \cup \cdots \cup C_{n}=\partial P$,

(2) $C_{0} \cap \cdots \cap C_{n}=\emptyset$.

Let $\Delta$ denote the standard $n$-simplex:

$$
\Delta=\left\{y=\left(y_{0}, \ldots, y_{n}\right) \in \mathbb{R}^{n+1} \mid y_{0}+\cdots+y_{n}=1,0 \leq y_{i} \leq 1\right\} .
$$

For each $1 \leq k \leq n$ the codimension $k$ faces of $\Delta$ are

$$
\Delta_{i_{1} \cdots i_{k}}=\left\{y \in \Delta \mid y_{i_{1}}=\cdots=y_{i_{k}}=0\right\} \text {, where } 0 \leq i_{1}<\cdots<i_{k} \leq n .
$$

Then every simplicial coloring $C=\left(C_{0}, \ldots, C_{n}\right)$ defines a map

$$
\psi_{C}: \mathcal{F}(\partial P) \rightarrow \mathcal{F}(\partial \Delta),
$$

given by $\psi_{C}(G)=\Delta_{i_{1} \cdots i_{k}}$ if $G$ belongs to $C_{i_{1}} \cap \cdots \cap C_{i_{k}}$ with $k$ maximal. Clearly $\psi_{C}$ respects the partial order.

\footnotetext{
${ }^{1}$ In fact, $\lambda=(-1)^{\frac{n(n+1)}{2}}$.
} 
Definition 1.11. Fix orientations of the polytope $P$ and the simplex $\Delta$. The combinatorial degree of a simplicial coloring $C=\left(C_{0}, \ldots, C_{n}\right)$ of $P$ is the combinatorial degree c.deg $\psi_{C}$ of the corresponding map $\psi_{C}: \mathcal{F}(\partial P) \rightarrow \mathcal{F}(\partial \Delta)$.

It follows from the definition that c.deg $\psi_{C}$ is alternating on the order of the $C_{i}$.

Recall that the normal fan $\Sigma_{P}$ of a polytope $P$ in $E$ is a complete fan in $E^{*}$ whose cones are

$$
\sigma_{G}=\left\{v \in E^{*} \mid\langle u, v\rangle \geq\left\langle u^{\prime}, v\right\rangle, \text { for all } u \in P, u^{\prime} \in G\right\},
$$

for every face $G$ of $P$. Note that the 1-dimensional cones (rays) of $\Sigma_{P}$ are generated by the inner normals to the facets of $P$. Thus, every coloring of $P$ defines a "coloring" of the rays of $\Sigma_{P}$ into $n+1$ colors. We arrive at the following definition.

Definition 1.12. Let $\Sigma$ be a fan in $\mathbb{R}^{n}$. A decomposition of the set $\Sigma(1)$ of rays of $\Sigma$ into a union of $n+1$ subsets

$$
\Sigma(1)=\Lambda_{0} \cup \cdots \cup \Lambda_{n}
$$

is called a coloring of $\Sigma$. We say a coloring is disjoint if the union above is disjoint.

A coloring is called simplicial if no (maximal) cone of $\Sigma$ contains rays of all $n+1$ colors, i.e. every set of rays $\left\{\rho_{i} \in \Lambda_{i}, 0 \leq i \leq n\right\}$ is not contained in any of the (maximal) cones of $\Sigma$.

Note that we allow multiple colors of a ray unless the coloring is disjoint.

Clearly, every simplicial coloring of a polytope $P$ defines a simplicial coloring of its normal fan $\Sigma_{P}$. Conversely, if $\Sigma$ is a projective fan (i.e. the normal fan of a polytope), then every simplicial coloring of $\Sigma$ defines a simplicial coloring of any polytope whose normal fan is $\Sigma$. It is not hard to see that these colorings will all have the same combinatorial degree (for example one can deduce it from Theorem [1.7). We will call it the combinatorial degree of the simplicial coloring of $\Sigma$.

Applying Theorem 1.7 we obtain an explicit formula for the combinatorial degree of a coloring of a fan $\Sigma$ in terms of complete flags of faces of $\Sigma$. For simplicity and for further purposes we will state this formula in the case when $\Sigma$ is simplicial (i.e. all cones of $\Sigma$ are simplicial) and the coloring is disjoint.

Fix $0 \leq i_{1}<\cdots<i_{k} \leq n$. Then we say that a cone $\sigma$ of $\Sigma$ is $\left(i_{1}, \ldots, i_{k}\right)$-colored if for every $1 \leq j \leq k$ the cone $\sigma$ contains a ray from $\Lambda_{i_{j}}$, and $k$ is maximal.

Let $\sigma$ be a maximal simplicial $\left(i_{1}, \ldots, i_{n}\right)$-colored cone for a simplicial disjoint coloring of $\Sigma$. We say it has positive orientation if a collection of generators $\left(e_{1}, \ldots, e_{n}\right)$, where $e_{j}$ generates the ray from $\Lambda_{i_{j}}$, gives a positive oriented frame for $\mathbb{R}^{n}$.

Corollary 1.13. Let $\Sigma$ be a projective simplicial fan in $\mathbb{R}^{n}$ and let

$$
\Sigma(1)=\Lambda_{0} \sqcup \cdots \sqcup \Lambda_{n}
$$

be a disjoint simplicial coloring. Fix any $k, 0 \leq k \leq n$. Then the combinatorial degree of the coloring is equal to $(-1)^{k}$ times the number of maximal $(0, \ldots, \widehat{k}, \ldots, n)$ colored cones, counted with orientations.

Now suppose that $\Sigma^{\prime}$ is a refinement of $\Sigma$ with the same set of rays. Then any simplicial coloring of $\Sigma$ is also a simplicial coloring of $\Sigma^{\prime}$. The following theorem shows that these colorings will have the same combinatorial degree. 
Theorem 1.14. Let $\Sigma$ and $\Sigma^{\prime}$ be projective fans such that $\Sigma^{\prime} \rightarrow \Sigma$ is a refinement and $\Sigma(1)=\Sigma^{\prime}(1)$. Consider a simplicial coloring of $\Sigma$. The induced coloring of $\Sigma^{\prime}$ is simplicial and has the same combinatorial degree.

Proof. The fact that the induced coloring of $\Sigma^{\prime}$ is simplicial follows by definition.

Let $P$ and $P^{\prime}$ be polytopes whose normal fans are $\Sigma$ and $\Sigma^{\prime}$, respectively. Furthermore, let $C$ (resp. $C^{\prime}$ ) be the simplicial coloring of $P\left(\right.$ resp. $P^{\prime}$ ) defined by the simplicial coloring of $\Sigma$ (resp. $\Sigma^{\prime}$ ). We need to show that c.deg $\psi_{C}=\operatorname{c.deg} \psi_{C^{\prime}}$.

We can assume that the origin lies in the interior of $P$ and $P^{\prime}$ and so the fans $\Sigma$ and $\Sigma^{\prime}$ consist of the cones over the proper faces of $P^{\circ}$ and $\left(P^{\prime}\right)^{\circ}$, respectively. By Proposition 1.9 it is enough to show that c.deg $\psi_{C}^{*}=\operatorname{c.deg} \psi_{C^{\prime}}^{*}$ for the dual maps.

Let $X$ denote the boundary of $P^{\circ}$. Projecting the proper faces of $\left(P^{\prime}\right)^{\circ}$ onto the faces of $X$ along the rays of $\Sigma$ we get a subdivision $X^{\prime}$ of $X$. It is clear that we get an order-preserving bijection between $\mathcal{F}\left(X^{\prime}\right)$ and the proper faces of $\left(P^{\prime}\right)^{\circ}$, and that the composition map $\psi^{\prime}: \mathcal{F}\left(X^{\prime}\right) \rightarrow \mathcal{F}\left(\partial\left(\Delta^{\circ}\right)\right)$ has the same combinatorial degree as $\psi_{C^{\prime}}^{*}$.

It remains to show that $\psi^{\prime}: \mathcal{F}\left(X^{\prime}\right) \rightarrow \mathcal{F}\left(\partial\left(\Delta^{\circ}\right)\right)$ refines $\psi_{C}^{*}: \mathcal{F}(X) \rightarrow \mathcal{F}\left(\partial\left(\Delta^{\circ}\right)\right)$. Indeed, $\psi_{C}^{*}$ sends an element $G \in \mathcal{F}(X)$ to $\Delta_{i_{1} \ldots i_{k}}^{*}$ if and only if the cone over $G$ is $\left(i_{1}, \ldots, i_{k}\right)$-colored. Suppose $G^{\prime} \in \mathcal{F}\left(X^{\prime}\right)$ is contained in $G$. Then the rays of the cone over $G^{\prime}$ are also rays of the cone over $G$ and, hence, will have the same colors $\left(i_{1}, \ldots, i_{k}\right)$ (all of them or fewer). Therefore, $\psi^{\prime}\left(G^{\prime}\right) \subset \Delta_{i_{1} \ldots i_{k}}^{*}$. By Proposition 1.4 c.deg $\psi^{\prime}=$ c.deg $\psi_{C}^{*}$, which completes the proof.

\section{TORIC RESIDUE}

Let $X=X(\Sigma)$ be an $n$-dimensional complete toric variety determined by a complete rational fan $\Sigma$ (see for example $[\mathrm{F}]$ ). Following the notation of $[\mathrm{F}]$ we let $N$ denote a lattice of rank $n$ and $N_{\mathbb{R}}$ the real vector space $N \otimes \mathbb{R}$ which contains the fan $\Sigma$. Also let $M=\operatorname{Hom}(N, \mathbb{Z})$ be the dual lattice and $M_{\mathbb{R}}$ the corresponding dual space $M \otimes \mathbb{R}$.

Let $\Sigma(1)=\left\{\rho_{1}, \ldots, \rho_{r}\right\}$ be the set of rays of $\Sigma$. Each ray $\rho_{i} \in \Sigma(1)$ determines a $\mathbb{T}$-invariant irreducible divisor $D_{i}$ on $X$. The variety $X$ has the homogeneous coordinate ring $S=\mathbb{C}\left[x_{1}, \ldots, x_{r}\right]$ graded by the Chow group $A_{n-1}(X)$ so that a monomial $x^{a}=\prod_{j=1}^{r} x_{j}^{a_{j}}$ has degree $\operatorname{deg}\left(x^{a}\right)=\left[\sum_{j=1}^{r} a_{j} D_{j}\right] \in A_{n-1}(X)$ (see C1). Denote by $S_{\alpha}$ the graded piece of $S$ consisting of all polynomials of degree $\alpha \in A_{n-1}(X)$. As shown in C1] $S_{\alpha}$ is canonically isomorphic to the global sections of the sheaf $\mathcal{O}_{X}(D)$ on $X$, where $\alpha=[D]$.

We will recall the definition of the toric residue C2 CCD. Consider $n+1$ homogeneous polynomials $F_{i} \in S_{\alpha_{i}}$, for $0 \leq i \leq n$. Their critical degree is defined to be

$$
\rho=\sum_{i=0}^{n} \alpha_{i}-\sum_{j=1}^{r} \operatorname{deg}\left(x_{j}\right)
$$

Then every polynomial $H$ of degree $\rho$ defines a meromorphic $n$-form on $X$ :

$$
\omega_{F}(H)=\frac{H \Omega}{F_{0} \cdots F_{n}},
$$

where $\Omega$ is the Euler form [CCD]. We use $F$ to denote the vector $\left(F_{0}, \ldots, F_{n}\right)$.

Suppose that $F_{i}$ do not vanish simultaneously on $X$. Then $X$ has an open cover $\mathcal{U}=\left\{U_{i}, 0 \leq i \leq n\right\}$, where $U_{i}=\left\{x \in X \mid F_{i}(x) \neq 0\right\}$. Therefore $\omega_{F}(H)$ defines a Čech cohomology class $\left[\omega_{F}(H)\right] \in H^{n}\left(X, \widehat{\Omega}_{X}^{n}\right)$, relative to the cover $\mathcal{U}$. Here $\widehat{\Omega}_{X}^{n}$ 
denotes the sheaf of Zariski $n$-forms on $X$. This class $\left[\omega_{F}(H)\right]$ is alternating on the order of the $F_{i}$ and is zero if $H$ belongs to the ideal $\left\langle F_{0}, \ldots, F_{n}\right\rangle$. The toric residue map

$$
\operatorname{Res}_{F}: S_{\rho} /\left\langle F_{0}, \ldots, F_{n}\right\rangle_{\rho} \rightarrow \mathbb{C}
$$

is given by $\operatorname{Res}_{F}(H)=\operatorname{Tr}_{X}\left(\left[\omega_{F}(H)\right]\right)$, where $\operatorname{Tr}_{X}$ is the trace map on $X$.

As proved in [CDD, when $X$ is simplicial the toric residue is in fact the sum of local Grothendieck residues:

Theorem 2.1 ( $[\mathrm{CCD}]$ ). Let $X$ be a complete simplicial toric variety. Let $F_{0}, \ldots, F_{n}$ be homogeneous polynomials which do not vanish simultaneously on $X$ and suppose the set $Z_{\hat{k}}=\left\{x \in X \mid F_{i}(x)=0,0 \leq i \leq n, i \neq k\right\}$ is finite, for some $k$. Then for any homogeneous polynomial $H$ of the critical degree $\rho$

$$
\operatorname{Res}_{F}(H)=(-1)^{k} \sum_{x \in Z_{\hat{k}}} \operatorname{Res}_{x}\left(\frac{\left(H / F_{k}\right) \Omega}{F_{0} \cdots \widehat{F}_{k} \cdots F_{n}}\right) .
$$

We consider a special case when $F_{i}=z_{i}$ are monomials whose product is the product of the variables, $z_{0} \cdots z_{n}=x_{1} \cdots x_{r}$. In this case the critical degree $\rho$ is zero and the toric residue is the multiplication by $\operatorname{Res}_{z}(1)$.

On the other hand, since the variables $x_{j}$ correspond to the rays $\rho_{j}$ of $\Sigma$, the monomials $z_{0}, \ldots, z_{n}$ define a disjoint coloring of $\Sigma$ :

$$
\Sigma(1)=\Lambda_{0} \sqcup \cdots \sqcup \Lambda_{n},
$$

where the ray $\rho_{j}$ lies in $\Lambda_{i}$ if and only if $x_{j}$ divides $z_{i}$. Clearly, this coloring is simplicial if and only if $z_{0}, \ldots, z_{n}$ do not vanish simultaneously on $X$.

In the following theorem we show that $\operatorname{Res}_{z}(1)$ is equal to the combinatorial degree of the coloring defined by $z_{0}, \ldots, z_{n}$.

Theorem 2.2. Let $X$ be an n-dimensional projective toric variety defined by a projective fan $\Sigma$. Let $z_{0}, \ldots, z_{n}$ be monomials in the homogeneous coordinate ring $S=\mathbb{C}\left[x_{1}, \ldots, x_{r}\right]$ such that

(1) $z_{0} \cdots z_{n}=x_{1} \cdots x_{r}$,

(2) $z_{0}, \ldots, z_{n}$ do not vanish simultaneously on $X$.

Then the toric residue $\operatorname{Res}_{z}(1)$ equals the combinatorial degree of the simplicial coloring of $\Sigma$ defined by $z_{0}, \ldots, z_{n}$.

Proof. First assume that $X$ is simplicial, and so $\Sigma$ is a simplicial fan. Let $Z_{i}$ be the zero locus of $z_{i}$ on $X$. Suppose the intersection $Z_{\hat{0}}=Z_{1} \cap \cdots \cap Z_{n}$ is infinite. Since it is a union of orbits it must contain a 1-dimensional orbit $O_{\tau}$, for some cone $\tau$ of dimension $n-1$. But this would imply that there are $n$ distinct irreducible components $Z_{i}^{\prime} \subset Z_{i}, 1 \leq i \leq n$, which contain $O_{\tau}$, i.e. the cone $\tau$ contains $n$ distinct rays of $\Sigma$, which is impossible when $\Sigma$ is simplicial. Therefore, $Z_{\hat{0}}$ is finite.

By Theorem 2.1 the toric residue is the sum of the Grothendieck residues over the points of $Z_{\hat{0}}$. But these points are the closed orbits $O_{\sigma}$ of $X$ that correspond to $(1, \ldots, n)$-colored maximal cones $\sigma$ of $\Sigma$. Therefore by Corollary 1.13 one only needs to check that the Grothendieck residue at $O_{\sigma}$ is equal to \pm 1 depending on the orientation of $\sigma$, which is straightforward.

In the general case let $X^{\prime}=X\left(\Sigma^{\prime}\right)$ be the toric variety determined by a simplicial refinement $\Sigma^{\prime}$ of $\Sigma$ with the same set of rays. Then $X$ and $X^{\prime}$ have the same homogeneous coordinate ring and the birational morphism $X^{\prime} \rightarrow X$ maps every $Z_{i}^{\prime}$ defined by $z_{i}$ on $X^{\prime}$ onto $Z_{i}$. By the functorial property of the trace map the toric 
residues $\operatorname{Res}_{z}(1)$ on $X$ and on $X^{\prime}$ are equal. On the other hand, by Theorem 1.14 the combinatorial degrees of the colorings of $\Sigma$ and $\Sigma^{\prime}$ are also equal, and the theorem follows.

We will now consider a more general case when $z_{i}$ are any (monic) monomials whose product is divisible by the product of the variables, $x_{1} \cdots x_{r} \mid z_{0} \cdots z_{n}$. In this case the quotient $z_{0} \cdots z_{n} / x_{1} \cdots x_{r}$ has the critical degree and it makes sense to consider the toric residue

$$
\operatorname{Res}_{z}\left(\frac{z_{0} \cdots z_{n}}{x_{1} \cdots x_{r}}\right)
$$

As before the monomials $z_{0}, \ldots, z_{n}$ define a coloring of $\Sigma$ which is now not necessarily disjoint. Theorem 2.2 implies the following more general statement.

Theorem 2.3. Let $X$ be an $n$-dimensional projective toric variety defined by a projective fan $\Sigma$. Let $z_{0}, \ldots, z_{n}$ be monomials in the homogeneous coordinate ring $S=\mathbb{C}\left[x_{1}, \ldots, x_{r}\right]$ such that

(1) $x_{1} \cdots x_{r} \mid z_{0} \cdots z_{n}$

(2) $z_{0}, \ldots, z_{n}$ do not vanish simultaneously on $X$.

Then the toric residue

$$
\operatorname{Res}_{z}\left(\frac{z_{0} \cdots z_{n}}{x_{1} \cdots x_{r}}\right)
$$

equals the combinatorial degree of the simplicial coloring of $\Sigma$ defined by $z_{0}, \ldots, z_{n}$.

Proof. To reduce our theorem to Theorem 2.2 we choose monomials $z_{0}^{\prime}, \ldots, z_{n}^{\prime}$ such that $z_{i}^{\prime} \mid z_{i}$ and $z_{0}^{\prime} \cdots z_{n}^{\prime}=x_{1} \cdots x_{r}$. Then

$$
\operatorname{Res}_{z^{\prime}}(1)=\operatorname{Res}_{z}\left(\frac{z_{0} \cdots z_{n}}{x_{1} \cdots x_{r}}\right) .
$$

Indeed, the open sets $U_{i}^{\prime}=\left\{z_{i}^{\prime}=0\right\}, 0 \leq i \leq n$, form a covering $\mathcal{U}^{\prime}$ of $X$. Also the covering $\mathcal{U}$ by the sets $U_{i}=\left\{z_{i}=0\right\}, 0 \leq i \leq n$, is a refinement of $\mathcal{U}^{\prime}$. Therefore the cocycles

$$
\left[\omega_{z^{\prime}}(1)\right] \in \mathcal{Z}^{n}\left(\mathcal{U}^{\prime}, \widehat{\Omega}_{X}^{n}\right) \quad \text { and } \quad\left[\omega_{z}\left(\frac{z_{0} \cdots z_{n}}{x_{1} \cdots x_{r}}\right)\right] \in \mathcal{Z}^{n}\left(\mathcal{U}, \widehat{\Omega}_{X}^{n}\right)
$$

define the same element in $H^{n}\left(X, \widehat{\Omega}_{X}^{n}\right)$.

Now it remains to show that the combinatorial degrees of the two colorings defined by $z_{0}, \ldots, z_{n}$ and $z_{0}^{\prime}, \ldots, z_{n}^{\prime}$ are the same. Let $P$ be a polytope whose normal fan is $\Sigma$, and let $C=\left(C_{0}, \ldots, C_{n}\right)$ and $C^{\prime}=\left(C_{0}^{\prime}, \ldots, C_{n}^{\prime}\right)$ be the two colorings of $P$ defined by $z_{0}, \ldots, z_{n}$ and $z_{0}^{\prime}, \ldots, z_{n}^{\prime}$, respectively. Consider $G \in \mathcal{F}(\partial P)$ and assume that $G$ belongs to $C_{i_{1}}^{\prime} \cap \cdots \cap C_{i_{k}}^{\prime}$ with $k$ maximal, where $0 \leq i_{1}<\cdots<i_{k} \leq n$. Then $G$ belongs to $C_{i_{1}} \cap \cdots \cap C_{i_{k}}$ and hence $\psi_{C}(G) \subset \psi_{C^{\prime}}(G)$. But this implies that $\psi_{C}$ refines $\psi_{C^{\prime}}$ and so they have the same combinatorial degree by Proposition 1.4

Remark 2.4. The statement in (2.1) also follows from the Global Transformation Law (Theorem 0.1 of CCD $)$.

\section{Monomial IDEAL}

In this section we study radical monomial ideals of the homogeneous coordinate ring $S$ of $X$. We answer the following question: When does a homogeneous polynomial $F \in S_{\alpha}$ of semiample degree $\alpha$ belong to a radical monomial ideal $I$ ? 
3.1. Semiample class. Let $D=\sum_{j=1}^{r} a_{j} D_{j}$ be a $\mathbb{T}$-invariant Cartier divisor on $X$. Then it determines a unique continuous function $\phi_{D}$ linear on each cone of $\Sigma$ such that $\phi_{D}\left(v_{j}\right)=-a_{j}, 1 \leq j \leq r$, where $v_{j}$ is the first lattice point along the ray $\rho_{j} \in \Sigma(1)$. Note that if $D$ and $D^{\prime}$ are linearly equivalent, then $\phi_{D}-\phi_{D}^{\prime}$ is a linear function on $N_{\mathbb{R}}$.

The divisor $D$ also determines a rational convex polytope $P_{D}$ in $M_{\mathbb{R}}$ :

$$
P_{D}=\left\{u \in M_{\mathbb{R}} \mid\left\langle u, v_{j}\right\rangle \geq-a_{j}, 1 \leq j \leq r\right\}=\left\{u \in M_{\mathbb{R}} \mid u \geq \phi_{D}\right\} .
$$

Linearly equivalent divisors have the same polytope up to a parallel translation.

Definition 3.1. A Cartier divisor $D$ is called semiample if the corresponding line bundle $\mathcal{O}_{X}(D)$ is generated by global sections. Also $\alpha=[D] \in A_{n-1}(X)$ is called a semiample class if $D$ is semiample.

A divisor $D$ is semiample if and only if the function $\phi_{D}$ is convex $([\vec{F}]$, Section 3.4).

In [M] A. Mavlyutov shows that if $\alpha$ is a semiample class on $X$, then there is a unique toric variety $X^{\prime}$ and a surjective morphism $\pi: X \rightarrow X^{\prime}$ such that $\alpha$ is the pull-back of an ample class $\alpha^{\prime}$ on $X^{\prime}$. Here we recall this construction.

Let $\alpha=[D]$ be a semiample class. First, with the polytope $P_{D}$ we associate a rational fan $\Sigma_{D}$ as follows. If $\operatorname{dim} P_{D}=n$, then $\Sigma_{D} \subset N_{\mathbb{R}}$ is the normal fan of $P_{D}$. In general let $L \subset N_{\mathbb{R}}$ be the orthogonal complement to the affine space spanned by $P_{D}$. Then for every face $G$ of $P_{D}$ the cone $\sigma_{G}$ contains $L$ (see (1.2)). Define $\Sigma_{D}$ to be the fan in $N_{\mathbb{R}} / L$ whose cones are the images $\left[\sigma_{G}\right]$ under the quotient map $N_{\mathbb{R}} \rightarrow N_{\mathbb{R}} / L$. The fan $\Sigma_{D}$ is rational with respect to the lattice $N / N \cap L$. Since $\Sigma_{D}$ is the same for all representatives $D$ of $\alpha$ we denote it by $\Sigma_{\alpha}$.

The quotient map $N \rightarrow N / N \cap L$ extends to a map of fans

$$
\tilde{\pi}: \Sigma \rightarrow \Sigma_{\alpha} .
$$

Indeed, for any maximal cone $\sigma \in \Sigma$ the restriction of $\phi_{D}$ to $\sigma$ defines a lattice point $u$ which is a vertex of $P_{D}$ (by convexity of $\phi_{D}$ ). Then $\tilde{\pi}(\sigma) \subset\left[\sigma_{u}\right]$. The map $\tilde{\pi}$ induces the surjective morphism

$$
\pi: X \rightarrow X^{\prime}, \quad \text { where } X^{\prime}=X\left(\Sigma_{\alpha}\right) .
$$

Note that when $\operatorname{dim} P_{D}=n$ the map $\tilde{\pi}: \Sigma \rightarrow \Sigma_{\alpha}$ is a refinement. Also $\Sigma=\Sigma_{\alpha}$ and $\tilde{\pi}=i d$ when $\alpha$ is an ample class.

3.2. Monomial ideal. Let us now consider a radical monomial ideal $I$ of $S$. We denote by $z_{1}, \ldots, z_{m}$ the minimal generating set of $I$. Similar to the construction of Section 3 these monomials determine a coloring of rays of the fan $\Sigma$ into $m$ colors: a ray $\rho_{j}$ has color $i$ if and only if $x_{j}$ divides $z_{i}$. Note that we allow multi-colored rays and not all the rays of $\Sigma$ are colored, in general.

Recall that the irrelevant ideal of $X$ is the ideal $B$ in $S$ generated by all the products $\prod_{\rho_{j} \notin \sigma} x_{j}$, where $\sigma$ runs over all (maximal) cones of $\Sigma$ (see [C1]). Similarly, we define the ideal $B_{\alpha}$ in $S$ generated by all the products $\prod_{\tilde{\pi}\left(\rho_{j}\right) \notin \sigma} x_{j}$, where $\sigma$ runs over all (maximal) cones of $\Sigma_{\alpha}$ and $\tilde{\pi}: \Sigma \rightarrow \Sigma_{\alpha}$ as above. Clearly, $B \subset B_{\alpha}$ and $B=B_{\alpha}$ if $\alpha$ is ample.

Remark 3.2. As mentioned in CCD], Section 2, for any ample degree $\alpha$ we have $S_{\alpha} \subset B$. This is not true in general, but as we will see in the proof of the next theorem, $S_{\alpha} \subset B_{\alpha}$ when $\alpha$ is semiample. 
The following is the main result of this section.

Theorem 3.3. Consider a radical monomial ideal $I=\left\langle z_{1}, \ldots, z_{m}\right\rangle$ of the homogeneous coordinate ring $S$ of $X$. Let $\alpha \in A_{n-1}(X)$ be semiample. The following are equivalent:

(1) $S_{\alpha} \subset I$,

(2) $F \in I$, where $F$ is a generic section of $\alpha$,

(3) $B_{\alpha} \subset I$,

(4) no (maximal) cone of $\Sigma_{\alpha}$ contains images of rays of $\Sigma$ of all $m$ colors,

(5) $\pi\left(Z_{1}\right) \cap \cdots \cap \pi\left(Z_{m}\right)=\emptyset$, where $\pi: X \rightarrow X^{\prime}$ is the morphism determined by $\tilde{\pi}: \Sigma \rightarrow \Sigma_{\alpha}$, and $Z_{i}$ is the zero locus of $z_{i}$ on $X$.

Proof. $(1) \Leftrightarrow(2)$ is clear.

$(2) \Leftrightarrow(3)$ Let $D=\sum_{j=1}^{r} a_{j} D_{j}$ be a representative of $\alpha$ and consider its polytope $P_{D}$. Since $F$ is generic, it is a linear combination with non-zero complex coefficients of the monomials $\chi^{u}$ as $u$ varies over the set $P_{D} \cap M$ ([F], Section 3.4). But $I$ is a monomial ideal, thus $F \in I$ if and only if every $\chi^{u}$ belongs to $I$.

In homogeneous coordinates $\chi^{u}=\prod_{j=1}^{r} x_{j}^{\left\langle u, v_{j}\right\rangle+a_{j}}$. From this you can see that every such monomial is divisible by some $\chi^{w}$, where $w$ is a vertex of $P_{D}$. Thus we can assume that $u$ varies over the vertices of $P_{D}$.

When $u$ is a vertex of $P_{D}$ we have $\left\langle u, v_{j}\right\rangle=-a_{j}$ if $\tilde{\pi}\left(\rho_{j}\right) \in \sigma_{u}$ and $\left\langle u, v_{j}\right\rangle>-a_{j}$ if $\tilde{\pi}\left(\rho_{j}\right) \notin \sigma_{u}$, where $\sigma_{u}$ is the cone of $\Sigma_{\alpha}$ corresponding to $u$. Therefore we can write

$$
\chi^{u}=\prod_{\tilde{\pi}\left(\rho_{j}\right) \notin \sigma_{u}} x_{j}^{\left\langle u, v_{j}\right\rangle+a_{j}},
$$

where all exponents are positive. Therefore $\chi^{u} \in I$ for all vertices $u$ if and only if for every maximal cone $\sigma$ of $\Sigma_{\alpha}$ there exists $k$ such that $z_{k} \mid \prod_{\tilde{\pi}\left(\rho_{j}\right) \notin \sigma} x_{j}$ (by (B.1) and the fact that in $z_{k}$ every variable has exponent 0 or 1 ). By the definition of $B_{\alpha}$ the latter is equivalent to $B_{\alpha} \subset I$.

$(4) \Leftrightarrow(5)$ Suppose there exists a maximal cone $\sigma^{\prime}$ in $\Sigma_{\alpha}$ which contains the images $\tilde{\pi}\left(\rho_{k_{1}}\right), \ldots, \tilde{\pi}\left(\rho_{k_{m}}\right)$, where $\rho_{k_{i}}$ has color $i$. It can be readily seen that the morphism $\pi: X \rightarrow X^{\prime}$ maps every orbit $O_{\tau}, \tau \in \Sigma$, onto the orbit $O_{\tau^{\prime}}$, where $\tau^{\prime}$ is the smallest cone of $\Sigma_{\alpha}$ containing $\tilde{\pi}(\tau)$, and hence it maps the closure $V(\tau)$ onto the closure $V\left(\tau^{\prime}\right)$. It follows then that for every $0 \leq i \leq n$ the image $\pi\left(V\left(\rho_{k_{i}}\right)\right)$ contains the closed orbit $O_{\sigma^{\prime}}$. Since $V\left(\rho_{k_{i}}\right)$ is an irreducible component of $Z_{i}$, we get a contradiction. The other implication is similar.

$(3) \Leftrightarrow(4)$ Before we give a proof let us take a closer look at the ideal $B_{\alpha}$.

We say that a collection of rays $\left\{\rho_{j_{1}}, \ldots, \rho_{j_{s}}\right\}$ of $\Sigma$ is primitive with respect to $\Sigma_{\alpha}$ if its image under $\tilde{\pi}$ does not lie in any of the cones of $\Sigma_{\alpha}$, but the image of every one of its proper subsets does. The corresponding collection of variables $\left\{x_{j_{1}}, \ldots, x_{j_{s}}\right\}$ will also be called primitive with respect to $\Sigma_{\alpha}$. The following is a slight generalization of the Batyrev description of the irrelevant ideal of the homogeneous coordinate ring.

Lemma 3.4. The irreducible components of the variety $V\left(B_{\alpha}\right)$ of the ideal $B_{\alpha}$ are the coordinate planes $V\left(x_{j_{1}}, \ldots, x_{j_{s}}\right)$ for each collections $\left\{x_{j_{1}}, \ldots, x_{j_{s}}\right\}$ primitive with respect to $\Sigma_{\alpha}$.

Proof. By definition the variety $V\left(B_{\alpha}\right)$ is the union of $V\left(x_{\hat{\sigma}}: \sigma \in \Sigma_{\alpha}\right)$, where for every maximal $\sigma \in \Sigma_{\alpha}$ we pick a variable $x_{\hat{\sigma}}$ whose corresponding ray is mapped 
outside of $\sigma$. Since the image of every primitive collection $\left\{\rho_{j_{1}}, \ldots, \rho_{j_{s}}\right\}$ does not lie in any of the cones of $\Sigma_{\alpha}$, the corresponding collection $\left\{x_{j_{1}}, \ldots, x_{j_{s}}\right\}$ appears among the collections $\left\{x_{\hat{\sigma}}: \sigma \in \Sigma_{\alpha}\right\}$. Therefore,

$$
\bigcup_{\substack{\left\{\rho_{j_{1}}, \ldots, \rho_{j_{s}}\right\} \\ \text { primitive }}} V\left(x_{j_{1}}, \ldots, x_{j_{s}}\right) \subseteq V\left(B_{\alpha}\right)
$$

On the other hand, every collection $\left\{x_{\hat{\sigma}}: \sigma \in \Sigma_{\alpha}\right\}$ contains a primitive one. (If a collection of rays whose image does not lie in any cone is not primitive, it contains a proper subset whose image does not lie in any cone, etc.) Thus,

$$
V\left(x_{\hat{\sigma}}: \sigma \in \Sigma_{\alpha}\right) \subseteq V\left(x_{j_{1}}, \ldots, x_{j_{s}}\right),
$$

for some primitive collection $\left\{\rho_{j_{1}}, \ldots, \rho_{j_{s}}\right\}$. The lemma follows.

Now we will finish the proof of the theorem. We have two irreducible decompositions:

$$
V(I)=\bigcup_{x_{k_{i}} \mid z_{i}} V\left(x_{k_{1}}, \ldots, x_{k_{m}}\right) \quad \text { and } \quad V\left(B_{\alpha}\right)=\bigcup_{\substack{\left\{\rho_{j_{1}}, \ldots, \rho_{j_{s}}\right\} \\ \text { primitive }}} V\left(x_{j_{1}}, \ldots, x_{j_{s}}\right) .
$$

Then $V(I) \subset V\left(B_{\alpha}\right)$ if and only if for every collection $\left\{x_{k_{1}}, \ldots, x_{k_{m}}\right\}$ with $x_{k_{i}} \mid z_{i}$, there exists a collection $\left\{x_{j_{1}}, \ldots, x_{j_{s}}\right\}$ primitive with respect to $\Sigma_{\alpha}$ such that

$$
V\left(x_{k_{1}}, \ldots, x_{k_{m}}\right) \subset V\left(x_{j_{1}}, \ldots, x_{j_{s}}\right),
$$

i.e., $\left\{\rho_{j_{1}}, \ldots, \rho_{j_{s}}\right\} \subset\left\{\rho_{k_{1}}, \ldots, \rho_{k_{m}}\right\}$. But the latter is equivalent to (4). Indeed, if $\left\{\rho_{k_{1}}, \ldots, \rho_{k_{m}}\right\}$ does not contain any primitive collection, then its image lies in some cone $\sigma$ of $\Sigma_{\alpha}$, i.e. $\sigma$ contains images of rays of all $m$ colors. The converse is also clear.

\section{Homogeneous polynomials of Residue one}

Let $X$ be a complete $n$-dimensional toric variety and let $F_{0}, \ldots, F_{n}$ be homogeneous polynomials of degrees $\alpha_{0}, \ldots, \alpha_{n}$, respectively. Consider the corresponding toric residue map

$$
\operatorname{Res}_{F}: S_{\rho} /\left\langle F_{0}, \ldots, F_{n}\right\rangle_{\rho} \rightarrow \mathbb{C} .
$$

As recently proved by Cox and Dickenstein [CD] this map is an isomorphism if the degrees $\alpha_{i}$ are semiample and have $n$-dimensional polytopes. Then the computation of the toric residue reduces to the problem of finding a homogeneous polynomial of residue one. This is an open problem. The results of the previous sections allow us to say something in this direction.

Assume $X$ is projective defined by a projective fan $\Sigma$. Let $\Sigma_{i}=\Sigma_{\alpha_{i}}$ be the fan associated with the degree $\alpha_{i}$ and let $\tilde{\pi}_{i}: \Sigma \rightarrow \Sigma_{i}$ be the corresponding map as in Section 3.1. We say that a disjoint simplicial coloring $\Sigma(1)=\Lambda_{0} \sqcup \cdots \sqcup \Lambda_{n}$ is compatible with $\Sigma_{0}, \ldots, \Sigma_{n}$ if it satisfies the condition of Theorem 3.3 for each $\Sigma_{i}$, i.e. no cone of $\Sigma_{i}$ contains images under $\tilde{\pi}_{i}$ of rays of all $n+1$ colors, for every $i$.

Corollary 4.1. Let $\alpha_{0}, \ldots, \alpha_{n}$ be semiample degrees on $X$ and let $\Sigma_{0}, \ldots, \Sigma_{n}$ be the corresponding fans. Fix any disjoint simplicial coloring of $\Sigma$ compatible with $\Sigma_{0}, \ldots, \Sigma_{n}$ :

$$
\Sigma(1)=\Lambda_{0} \sqcup \cdots \sqcup \Lambda_{n}
$$


Let $z_{i}=\prod_{\rho_{j} \in \Lambda_{i}} x_{j}$ be squarefree monomials, $0 \leq i \leq n$. Then for any homogeneous polynomials $F_{0}, \ldots, F_{n}$ of degrees $\alpha_{0}, \ldots, \alpha_{n}$ there are homogeneous polynomials $A_{i j}$ such that

$$
F_{i}=A_{i 0} z_{0}+\cdots+A_{\text {in }} z_{n}, \quad 0 \leq i \leq n .
$$

Furthermore,

$$
\operatorname{Res}_{F}(\operatorname{det}(A))=\operatorname{Res}_{z}(1)=\operatorname{c.deg}(\Lambda),
$$

where $\operatorname{cog}(\Lambda)$ is the combinatorial degree of the coloring above.

Proof. The existence of $A_{i j}$ is a consequence of Theorem 3.3 The first statement in (4.1) follows from the Global Transformation Law (Theorem 0.1, CCD]) and the second statement follows from Theorem 2.2.

Notice that if the degrees are ample, then $\Sigma=\Sigma_{0}=\cdots=\Sigma_{n}$ and we obtain many different ways of constructing a polynomial of residue one. In fact, every choice of a disjoint simplicial coloring of $\Sigma$ of combinatorial degree one gives rise to such a polynomial. This extends previously known constructions by D'Andrea and Khetan $[\mathrm{AK}]$ and Cattani, Cox and Dickenstein $\mathrm{CCD}$. Also, when the degrees are the same $\left(\Sigma_{0}=\cdots=\Sigma_{n}\right)$ there are many ways of choosing a compatible coloring of combinatorial degree one. It is not true, however, that compatible colorings of combinatorial degree one exist for any collection of $n+1$ semiample degrees. The simplest counterexample is presented in Figure 1
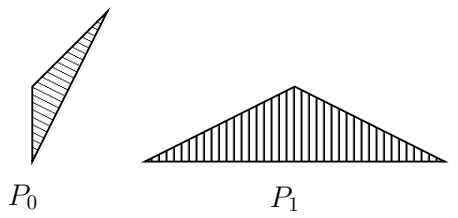
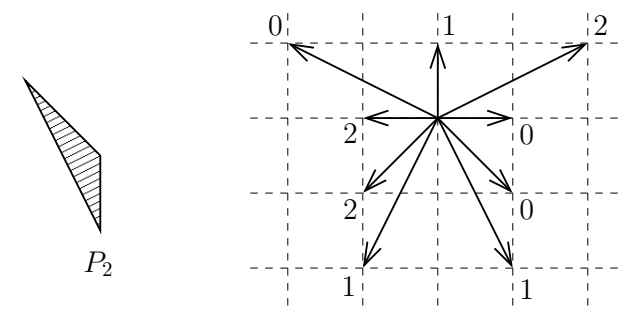

FiguRE 1.

Here $P_{0}, P_{1}$ and $P_{2}$ are the polytopes of semiample degrees $\alpha_{0}, \alpha_{1}$ and $\alpha_{2}$ on the toric surface determined by the complete fan $\Sigma$ on the right. The rays labeled with $i$, for $i=0,1,2$, form the fan $\Sigma_{i}$ corresponding to $\alpha_{i}$. One can check that there are no compatible colorings of $\Sigma$ of combinatorial degree one. In the forthcoming paper KS further methods are developed to deal with examples like that (in fact, all twodimensional examples and a new class of $n$-dimensional examples are considered).

\section{ACKNOWLEDGMENTS}

I thank Eduardo Cattani for introducing me to the subject, his constant attention to this work, and numerous discussions. I am also grateful to David Cox for helpful discussions, useful comments, and a suggestion on how to improve the result of Theorem [3.3. The result of Theorem[2.2 was presented at the MEGA 2003 conference in Kaiserslautern. 


\section{REFERENCES}

[AK] C. D'Andrea, A. Khetan, Macaulay style formulas for toric residues, preprint math.AG/0307154.

[BM] V. Batyrev, E. Materov, Toric Residues and Mirror Symmetry, Moscow Math. J. 2 (2002), no. 3, 435-475. MR.1988969

[CCD] E. Cattani, D. Cox, A. Dickenstein, Residues in Toric Varieties, Compositio Math. 108 (1997), no. 1, 35-76. MR1458757 (98f:14039)

[CaD] E. Cattani, A. Dickenstein, A global view of residues in the torus, J. Pure Appl. Algebra 117/118 (1997), 119-144. MR 1457836 (98i:14050)

[CD] D. Cox, A. Dickenstein, A Codimension Theorem for Complete Toric Varieties, preprint, math.AG/0310108.

[CDS] E. Cattani, A. Dickenstein, B. Sturmfels, Residues and Resultants, J. Math. Sci. Univ. Tokyo 5 (1998), 119-148. MR1617074 (2000b:14065)

[C1] D. A. Cox, The homogeneous coordinate ring of a toric variety, J. Algebr. Geom. 4 (1995), 17-50. MR 1299003 (95i:14046)

[C2] D. A. Cox, Toric residues, Arkiv für Matematik 34 (1996) 73-96. MR1396624 (97e:14062)

[F] W. Fulton, Introduction to Toric Varieties, Princeton Univ. Press, Princeton, 1993. MR.1234037 (94g:14028)

[GKh1] O. A. Gelfond and A. G. Khovanskii, Newton polyhedra and Grothendieck residues (in Russian), Dokl. Akad. Nauk, 350, no. 3 (1996), 298-300. MR1444043 (98b:32004)

[GKh2] O. A. Gelfond, A. G. Khovanskii, Toric geometry and Grothendieck residues, Moscow Math. J. 2 (2002), no. 1, 99-112. MR 1900586 (2003h:14074)

[KS] A. Khetan, I. Soprounov, Partition matrices for polytopes towards computing toric residue, preprint, math.AG/0406279.

[Kh] A. G. Khovanskii, Newton polyhedra, a new formula for mixed volume, product of roots of a system of equations, Fields Inst. Comm., Vol. 24 (1999), 325-364. MR1733583 (2001d:52013)

[M] A. Mavlyutov, The Hodge structure of semiample hypersurfaces and a generalization of the monomial-divisor mirror map, Advances in algebraic geometry motivated by physics, Contemp. Math. 276 (2000). MR1837119 (2002c:14067)

[S] I. Soprounov, Residues and tame symbols on toroidal varieties, to appear in Compositio Math., math.AG/0203114.

Department of Mathematics and Statistics, University of Massachusetts, Amherst, MASSAChUSETTS 01003

E-mail address: isoprou@math.umass.edu 